Characterizing counter-streaming interpenetrating plasmas relevant to astrophysical collisionless shocks

J. S. Ross, P. Amendt, D. Berger, L. Divol, S. H. Glenzer, N. Kugland, C. Plachety, B. Remington, D. Ryutov, D. H. Froula, C. Sorce, G.

Fiskel, W. Rozmus, Y. Kuramitsu, H. Takabe, T. Morita, A. Spitkovsky, C. Kuranz, M. Grosskopf, P. Drake, G. Gregori, J. Meineke, M. Koenig, A. Ravasio, A. Pelka, T. Vinci, E. Liang, R. Presura, H. -S Park

December 15, 2011

Physics of Plasma 
This document was prepared as an account of work sponsored by an agency of the United States government. Neither the United States government nor Lawrence Livermore National Security, LLC, nor any of their employees makes any warranty, expressed or implied, or assumes any legal liability or responsibility for the accuracy, completeness, or usefulness of any information, apparatus, product, or process disclosed, or represents that its use would not infringe privately owned rights. Reference herein to any specific commercial product, process, or service by trade name, trademark, manufacturer, or otherwise does not necessarily constitute or imply its endorsement, recommendation, or favoring by the United States government or Lawrence Livermore National Security, LLC. The views and opinions of authors expressed herein do not necessarily state or reflect those of the United States government or Lawrence Livermore National Security, LLC, and shall not be used for advertising or product endorsement purposes. 


\title{
Characterizing counter-streaming interpenetrating plasmas relevant to astrophysical collisionless shocks
}

J. S. Ross ${ }^{1}$, S. H. Glenzer ${ }^{1}$, P. Amendt ${ }^{1}$, R. Berger ${ }^{1}$, L. Divol ${ }^{1}$, N. L. Kugland ${ }^{1}$, C. Plechaty ${ }^{1}$, B. Remington ${ }^{1}$, D. Ryutov $^{1}$, W. Rozmus ${ }^{2}$, D. H. Froula ${ }^{3}$, G. Fiksel ${ }^{3}$, C. Sorce ${ }^{3}$, Y. Kuramitsu ${ }^{4}$, T. Morita ${ }^{4}$, Y. Sakawa ${ }^{4}$, H. Takabe $^{4}$, P. Drake ${ }^{5}$, M. Grosskopf ${ }^{5}$, C. Kuranz ${ }^{5}$, G. Gregori ${ }^{6}$, J. Meinecke ${ }^{6}$, C. D. Murphy ${ }^{6}$, M. Koenig ${ }^{7}$,

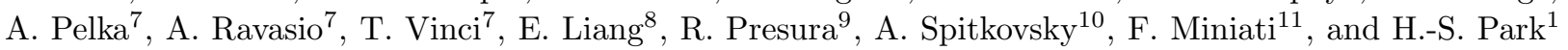

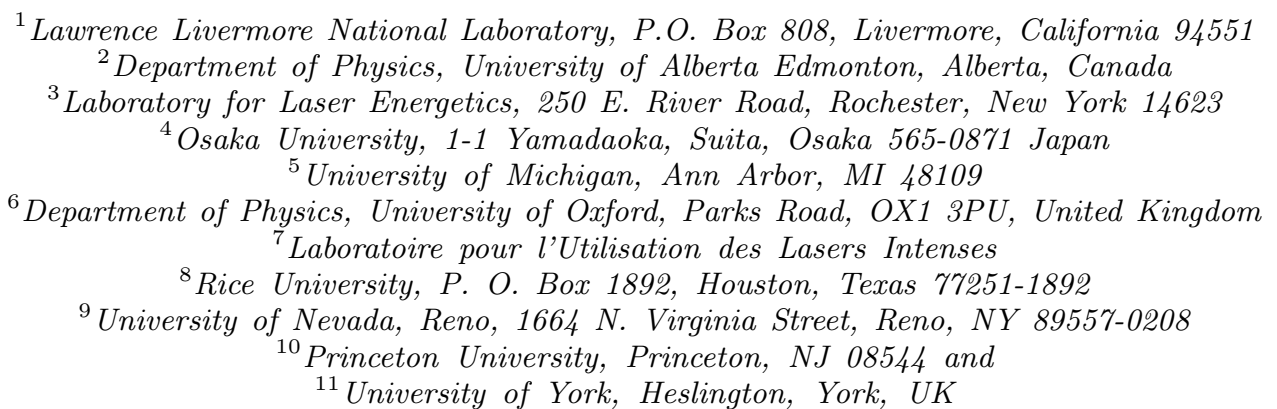

\begin{abstract}
A series of Omega experiments have produced and characterized high velocity counter-streaming plasma flows relevant for the creation of collisionless shocks. Single and double $\mathrm{CH}_{2}$ foils have been irradiated with a laser intensity of $\sim 10^{16} \mathrm{~W} / \mathrm{cm}^{2}$. The laser ablated plasma was characterized 4 $\mathrm{mm}$ from the foil surface using Thomson scattering. A peak plasma flow velocity of $2,000 \mathrm{~km} / \mathrm{s}$, an electron temperature of $\sim 110 \mathrm{eV}$, an ion temperature of $\sim 30 \mathrm{eV}$, and a density of $\sim 10^{18} \mathrm{~cm}^{-3}$ were measured in the single foil configuration. Significant increases in electron and ion temperatures were seen in the double foil geometry. The measured single foil plasma conditions were used to calculate the ion skin depth, $\mathrm{c} / \omega_{p i} \sim 0.16 \mathrm{~mm}$, the interaction length, $\ell_{i n t}$, of $\sim 8 \mathrm{~mm}$, and the Coulomb mean free path, $\lambda_{m f p} \sim 27 \mathrm{~mm}$. With $\mathrm{c} / \omega_{p i} \ll \ell_{i n t}<\lambda_{m f p}$ we are in a regime where collisionless shock formation is possible.
\end{abstract}

\section{INTRODUCTION}

Shocks in astrophysics are ubiquitous, occurring in supernovae, gamma ray bursts, and protostellar jets. In a broad range of low density astrophysical plasmas, the ion-ion collision mean free path is typically very large compared to the relevant spatial scales. Hence, when shocks form they are typically collisionless, resulting from plasma instabilities and self-generated magnetic fields [1, 2]. High power laser experiments can achieve the conditions necessary for the formation of collisionless shocks [3], allowing laboratory studies of this unique plasma instability physics. Our experiments are aimed at probing the importance of the electromagnetic (Weibel) [4] and electrostatic instability formation in collisionless shocks.

To generate collisionless shocks in the laboratory $[5,6]$ a number of criteria must be fulfilled. The interaction must be collisionless; for our experimental geometry this can be expressed in terms of the Coulomb mean free path, $\lambda_{m f p}$, between counter-streaming flows,

$$
\lambda_{m f p}=5 \times 10^{-13} \frac{A_{z}^{2}}{Z^{4}} \frac{\mathbf{v}^{4}}{n_{z}}
$$

where $A_{z}$ is the ion mass in amu, $Z$ is the average charge state, $\mathbf{v}$ is the velocity of each flowing plasma at the location of interest, and $n_{z}$ is the ion density of each flowing plasma stream. The Coulomb mean free path must be greater than the interaction length, $\ell_{i n t}$, which in turn must be greater than the width of the shock transition region $\left(\ell^{*}\right)$. If these conditions are achieved collisionless shock formation is possible but by no means guaranteed.

The width of the shock transition region has been investigated for both electrostatic and electromagnetic instabilities $[7,8]$. For the electrostatic case the width of the shock transition region is,

$$
\ell_{E S}^{*}=K^{(1)} \frac{\mathbf{v}}{\omega_{p i}} \frac{W}{T_{e}}
$$

where $W(\mathrm{eV})=5.2 \times 10^{-13} A_{z}[\mathbf{v}(\mathrm{cm} / \mathrm{s})]^{2}$ is the kinetic energy per ion, $\omega_{p i}$ is the ion plasma frequency, $T_{e}$ is the electron temperature and $K^{(1)}>>1$ is a numerical factor accounting for the number of growth lengths required for the instability to fully develop. Numerical simulations have estimated this factor to be $K^{(1)}=30$ for electrostatic shock formation [8]. In the case of electromagnetic instabilities the width of the transition region can be written,

$$
\ell_{E M}^{*}=K^{(2)} \frac{c}{\omega_{p i}}
$$

where $c$ is the speed of light and $K^{(2)}$ is analogous to $K^{(1)}$ but determined for electromagnetic instabilities. Again, based on numerical simulations, $K^{(2)}=100$ [9]. 
A pair of foils irradiated by high energy laser beams are used to generate high-velocity, counter-streaming plasma flows $[10,11]$. Thomson scattering $[12-14]$ is used to characterize the laser-produced $[15,16]$ plasma by fitting the measured data with the Thomson scattering dynamic structure factor, $S(\mathbf{k}, \omega)$. Assuming Maxwellian velocity distributions, the dynamic structure factor is,

$$
\frac{|\epsilon|^{2}}{2 \pi} S(\mathbf{k}, \omega)=\left|1+\chi_{i}\right|^{2} F_{e}\left(\frac{\omega}{k}\right)+\left|\chi_{e}\right|^{2} \sum_{j \in \text { ions }} \frac{n_{j}}{n_{e}} Z_{j}^{2} F_{j}\left(\frac{\omega}{k}\right)
$$

where $\omega$ is the frequency of the scattering wave, $Z_{j}$ is the charge state of ion species $j, n_{e}$ is the electron density, $n_{j}$ is the ion density of species $j, \epsilon=1+\chi_{i}+\chi_{e}$, $\chi_{i}$ and $\chi_{e}$ are the ion and electron susceptibility respectively, $F_{j} \equiv \int d^{3} v f_{j}(\vec{v}) \delta(\omega+\vec{k} \cdot \vec{v}), f_{e}$ and $f_{j}$ are the Maxwellian distributions for electrons and ion species $j$ respectively, $\vec{k}=\vec{k}_{s}-\vec{k}_{0}, \vec{k}_{o}$ is the wave number of probe beam, and $\vec{k}_{s}$ is the wave number of the scattered light. A sum of shifted Maxwellian ion velocity distributions for each species are used for the double foil configuration to account for the counter-streaming ion flows when calculating $S(\mathbf{k}, \omega)$. The electrons are assumed to be a single Maxwellian for both configurations due to electronelectron mean free path of $\sim 20 \mu \mathrm{m}$ or less for our conditions. The complete Thomson scattering spectrum, scattering from high frequency fluctuations (electron feature) and low frequency fluctuations (ion feature), is measured. The electron temperature and density, the ion temperature $\left(T_{i}\right)$, and the plasma flow velocity are then determined with high accuracy by comparing the Thomson scattering cross section, calculated using Eq. 4, to the scattered spectra. Then using the measured plasma conditions the likelihood of collisionless shock formation is assessed.

\section{EXPERIMENT}

\section{A. Target Configuration}

A single or pair of $\mathrm{CH}_{2}$ foils are positioned $4 \mathrm{~mm}$ from the target chamber center (TCC) as shown in Figure 1. Each foil is $2 \mathrm{~mm}$ in diameter and $0.5 \mathrm{~mm}$ in thickness. They are heated with 10, $351 \mathrm{~nm}$, laser beams. Each beam delivers $500 \mathrm{~J}$ in a 1 ns square pulse. The beams use distributed phase plates to produce supergaussian focal spots with a supergaussian exponent of 4.3 and a diameter of $\sim 250 \mu \mathrm{m}$. This results in an overlapped laser intensity of $\sim 10^{16} \mathrm{~W} / \mathrm{cm}^{2}$.

A $527 \mathrm{~nm}$ laser beam is used as a Thomson scattering probe. The probe beam is focused at TCC and has a 70 $\mu \mathrm{m}$ diameter focal spot and a pulse length of $1 \mathrm{~ns}$. A total probe energy of $40 \mathrm{~J}$ was used. The Thomson scattered light is collected $116.8^{\circ}$ relative to the probe resulting in a probed k-vector normal to the target surface. A second target configuration, rotated by $90^{\circ}$, is also used which

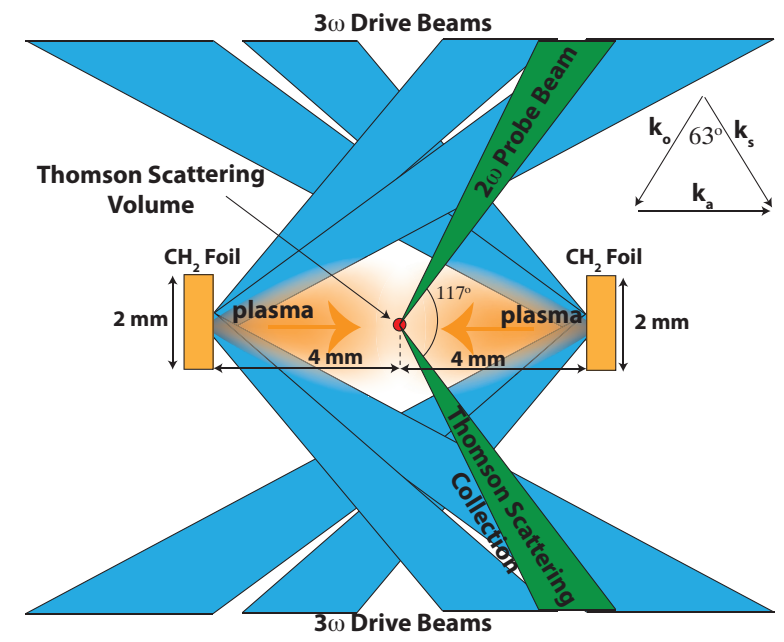

FIG. 1: (Color online) The experimental setup is shown for the double foil configuration. Each foil is irradiated with 10, $351 \mathrm{~nm}(3 \omega)$, laser beams using $1 \mathrm{~ns}$ square pulses with 250 $\mu \mathrm{m}$ focal spots. A $527 \mathrm{~nm}(2 \omega)$ probe beam is focused at the target chamber center. Thomson scattered light is collected $117^{\circ}$ relative to the probe. This Thomson scattering geometry results in a matched k-vector normal to the target surface.

results in a probed k-vector parallel to the target surface.

\section{B. Thomson scattering diagnostic}

The Thomson scattered light of the probe laser in the plasma is collected with an achromatic fused silica $\mathrm{f} / 10$ lens with a focal length of $50 \mathrm{~cm}$. The optic is mounted at a distance of $50 \mathrm{~cm}$ from the plasma. The angle between the collection optic and the input port of the TS probe is $116.8^{\circ}$. A fused silica blast shield is mounted before the collection optic. The collimated light is then transported by a series of turning mirrors to a 1-meter spectrometer and a $1 / 3$-meter spectrometer located 3 meters from the target chamber. The 1-meter spectrometer is used to measure the ion feature and the $1 / 3$-meter spectrometer measures the electron feature. A $50 \%$ reflectivity aluminum mirror, used to minimize wavelength sensitivity, splits the scattered signal between the two spectrometers. A $7.5 \mathrm{~cm}$ focusing mirror with a $75 \mathrm{~cm}$ focal length images the scattered light onto the entrance slit of the $1 \mathrm{~m}$ spectrometer with a magnification of 1.5:1. The spectrometer uses a 3600 lines/mm grating and a $200 \mu \mathrm{m}$ entrance slit. A Hamamatsu 7700 streak camera using a 5 ns sweep window is coupled to the output of each spectrometer. A $7.5 \mathrm{~cm}$ focusing mirror with a $45 \mathrm{~cm}$ focal length images onto the entrance slit of the $1 / 3$-meter spectrometer with a magnification of 0.9:1. The $1 / 3$-meter spectrometer uses a 150 lines/mm grating and a $100 \mu \mathrm{m}$ entrance slit. The Thomson scattering volume is defined by the overlap of both slit images, the streak camera slit and the spectrometer slit in the plasma $(130 \mu \mathrm{m} \times 130 \mu \mathrm{m}$ for the 1 -meter system and $110 \mu \mathrm{m} \times 110 \mu \mathrm{m}$ for the $1 / 3$-meter 

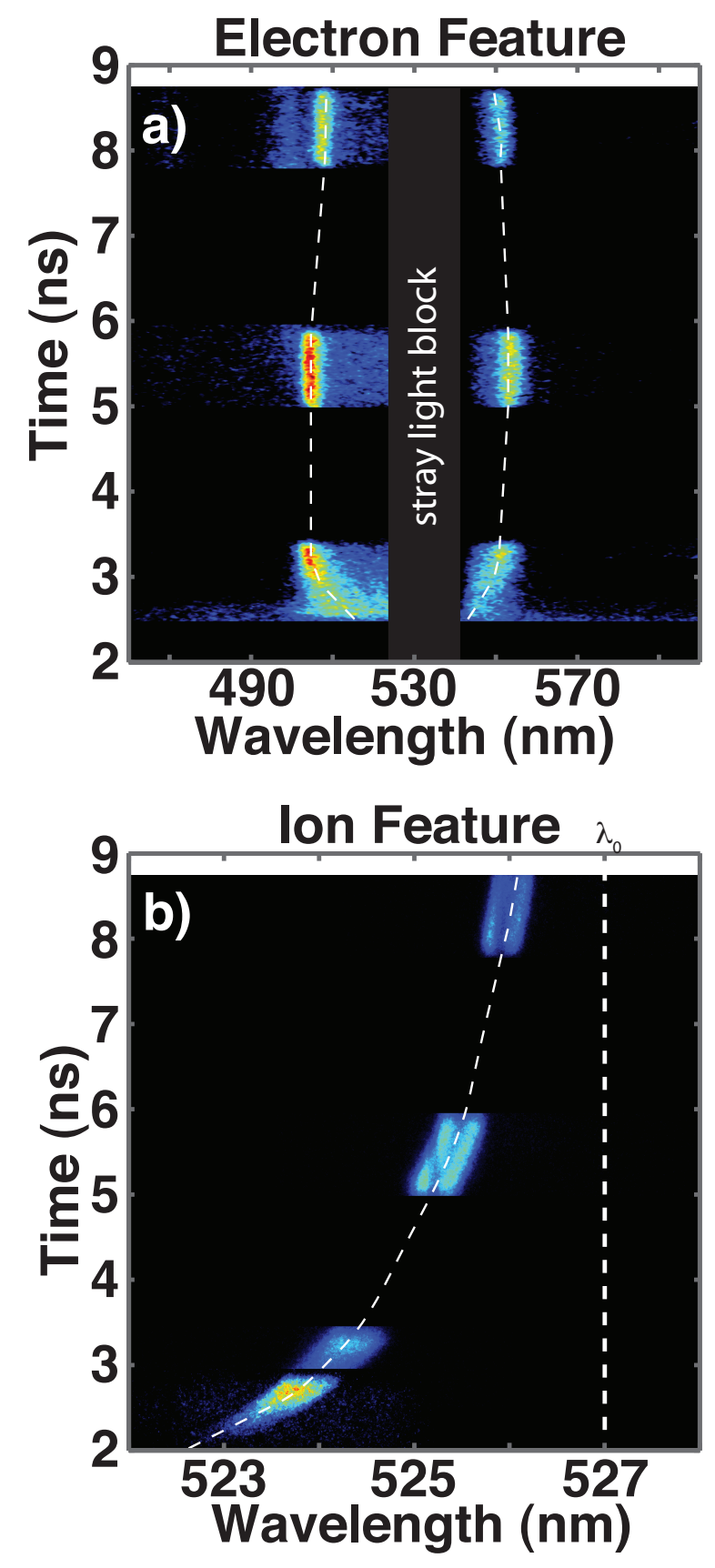

FIG. 2: (Color Online) A composite image is shown of the electron feature (a), and the ion feature (b) for the single foil configuration. A heavy dashed line in (b) is shown at the wavelength of the Thomson scattering probe beam. Thin dashed lines are shown to guide the eye.

system), with the probe beam (70 $\mu$ m diameter).

\section{RESULTS}

Figure 2 shows the composite Thomson scattering data for a series of single foil measurements with different
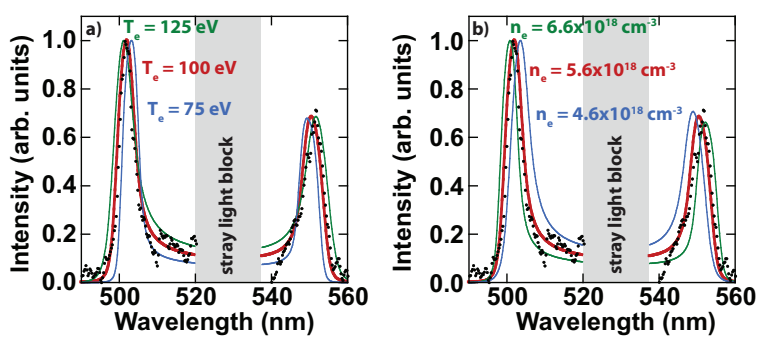

FIG. 3: (Color) The Thomson scattering cross section is fit to the measured Thomson scattering electron feature at 5.5 ns to determine the electron temperature and density from a single foil experiment. The best fit to the experimental data (red line) is calculated using an electron temperature of 100 $\mathrm{eV}$ and an electron density of $5.6 \times 10^{18} \mathrm{~cm}^{-3}$. a) The electron temperature is increased to $125 \mathrm{eV}$ (green line) and decreased to $75 \mathrm{eV}$ (blue line) to demonstrate the sensitivity of the fit. b) The electron density is varied from $6.6 \times 10^{18} \mathrm{~cm}^{-3}$ (green line) to $4.6 \times 10^{18} \mathrm{~cm}^{-3}$ (blue line) as well. A stray light block is used and heavily filters wavelengths between $520-537 \mathrm{~nm}$.

probe beam timings ranging from a beam delay of 2.0 ns to $8.8 \mathrm{~ns}$. The electron temperature and density are determined from the electron feature [Fig. 2 (a)]. The wavelength separation between the observed peaks is dominated by the electron density and the width of the observed peaks is a measure of the electron temperature. Plasma flow velocities in the range of $1.8 \times 10^{8} \mathrm{~cm} / \mathrm{s}$ to $4.0 \times 10^{7} \mathrm{~cm} / \mathrm{s}$ are measured from the ion feature's [Fig. 2 (b)] Doppler shift relative to the incident probe wavelength. The ion feature is also used to measurement the ion temperature. Both carbon and hydrogen are assumed to be fully ionized for all target configurations due to the measured electron temperature.

The experimental data from Figure 2 at $5.5 \mathrm{~ns}$ is compared to the Thomson scattering cross section (using Eq. 4) in Figures 3 and 4. Combined electron and ion feature fitting results provide the plasma state parameters of $\mathbf{v}$, $n_{e}, T_{e}$ and $T_{i}$. We estimate the errors on these parameters by studying the sensitivity to the fits: $\pm 10 \%$ in $\mathbf{v}$, $\pm 15 \%$ in $n_{e}, \pm 15 \%$ in $T_{e}$, and $\pm 20 \%$ in $T_{i}$.

Figure 5 shows the Thomson scattering data from the double foil target configuration. The electron feature from the double foil configuration is shown in Figure 5 (a). Again the width of the electron feature is a measure of the electron temperature which is clearly higher than the temperature in the single foil configuration. The electron density has also increased relative to the single foil configuration as expected. The spectral shift of the ion feature [Fig. 5 (a)] is very similar to that of the single foil configuration early in time, but after $5 \mathrm{~ns}$ a smaller spectral shift is observed in the double foil data compared to the single flow data. This is a results of a decreasing plasma flow velocity for the counter-streaming flows configuration late in time. The observed ion feature spectra are also significantly broader than those of the single foil data. The increased width of the ion feature is a function of increasing ion temperature. The red-shifted feature is 

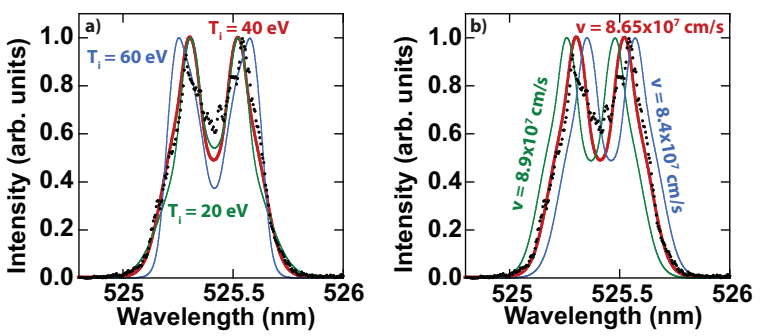

FIG. 4: (Color) The Thomson scattering cross section is fit to the measured Thomson scattering ion feature at $5.5 \mathrm{~ns}$ to determine the ion temperature and plasma flow velocity. The best fit to the experimental data (red line) is calculated using an electron temperature and density determined from the electron feature $\left(100 \mathrm{eV}\right.$ and $\left.5.6 \times 10^{18} \mathrm{~cm}^{-3}\right)$, and ion temperature of $40 \mathrm{eV}$ and a plasma flow velocity of $8.65 \times 10^{7}$ $\mathrm{cm} / \mathrm{s}$. a) The ion temperature is increased to $60 \mathrm{eV}$ (green line) and decreased to $20 \mathrm{eV}$ (blue line) to demonstrate the sensitivity of the fit. b) The plasma flow velocity is varied from $8.9 \times 10^{7} \mathrm{~cm} / \mathrm{s}$ (green line) to $8.4 \times 10^{7} \mathrm{~cm} / \mathrm{s}$ (blue line) as well.

also visible in the double foil late in time. Early in time the spectral field of view prevents the measurement of both blue-shifted and red-shifted ion features simultaneously in the double foil configuration.

The double foil Thomson scattering measurements are compared to the Thomson scattering cross section in Figures 6 and 7 to determine plasma conditions and estimate the error in the measurements. Similar errors to the single foil data are found for the double foil data: $\pm 10 \%$ in $\mathbf{v}, \pm 15 \%$ in $n_{e}, \pm 15 \%$ in $T_{e}$, and $\pm 20 \%$ in $T_{i}$.

Thomson scattering measurements are also made with a k-vector parallel to the target surface, orthogonal to the k-vector used in the configurations shown in Figures 2 and 5 . The a composite image of the ion feature for this parallel k-vector is shown in Figure 8 (a). The ion feature is nearly centered around the incident probe wavelength of $527 \mathrm{~nm}$, an indication that there is minimal plasma flow velocity along the match k-vector parallel to the target surface. This orthogonal k-vector allows an independent measurement of the ion temperature and an assessment of the ion temperature isotropy. The ion temperature measured with a k-vector perpendicular and parallel to the target surface is shown in Figure 8 (b). There is no measurable difference between these ion temperatures.

The Thomson scattering form factor is fit to the measured spectrum to determine the plasma flow velocity, the electron density, the electron temperature and the ion temperature (Figure 9). A plasma flow velocity [Fig. 9 (a) $]$ of $1.85 \times 10^{8} \pm 0.19 \times 10^{8} \mathrm{~cm} / \mathrm{s}$ is measured at $2.5 \mathrm{~ns}$ for both the single foil and double foil configurations. As the plasma continues to evolve the single foil data flow velocity decreases to $5.25 \times 10^{7} \pm 0.53 \times 10^{7} \mathrm{~cm} / \mathrm{s}$ at $8.8 \mathrm{~ns}$. The double foil data decreases more rapidly to a velocity of $3.95 \times 10^{7} \pm 0.40 \times 10^{7} \mathrm{~cm} / \mathrm{s}$ at the same time, which we atrribute to interactions between the counter-streaming plasma flows.
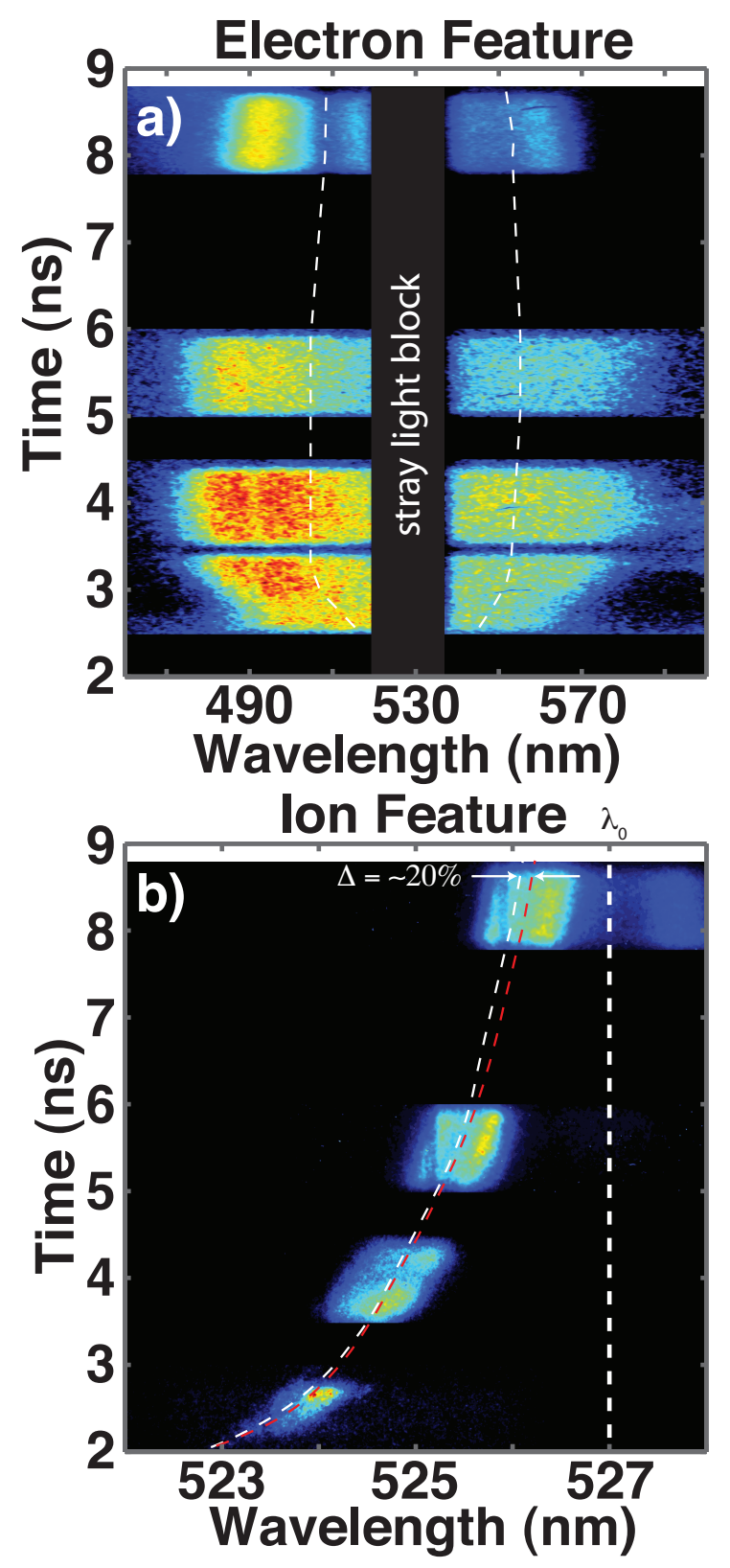

FIG. 5: (Color online) A composite image is shown of the electron feature (a), and the ion feature (b) for the double foil configuration. A heavy dashed line is shown at the wavelength of the Thomson scattering probe beam. The thin white dashed lines from Figure 2 are reproduced to facilitate comparisons of the spectral shifts. The thin red dashed line is a guide the eye for the double foil spectra. A decrease in spectral shift of $\sim 20 \%$ relative to the single foil data is observed at $8.8 \mathrm{~ns}$, a result of decreasing plasma flow velocity.

The electron density [Fig. 9(b)] is measured for the single and double foil configurations. A factor of two increase in the electron density is seen comparing the single foil and double foil configurations, an indication of collisionless interpenetration. If the interaction was dominated by collisions, stagnation would be expected, 

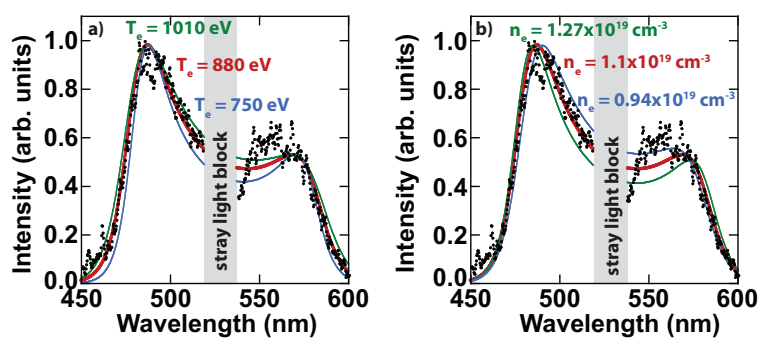

FIG. 6: (Color) The Thomson scattering cross section is fit to the measured Thomson scattering electron feature at 5.5 ns to determine the electron temperature and density from a double foil experiment. The best fit to the experimental data (red line) is calculated using an electron temperature of $880 \mathrm{eV}$ and an electron density of $1.1 \times 10^{19} \mathrm{~cm}^{-3}$. a) The electron temperature is increased to $1010 \mathrm{eV}$ (green line) and decreased to $750 \mathrm{eV}$ (blue line) to demonstrate the sensitivity of the fit. b) The electron density is varied from $1.27 \times 10^{19}$ $\mathrm{cm}^{-3}$ (green line) to $0.94 \times 10^{19} \mathrm{~cm}^{-3}$ (blue line) as well. A stray light block is used and heavily filters wavelengths between 520 - $537 \mathrm{~nm}$.
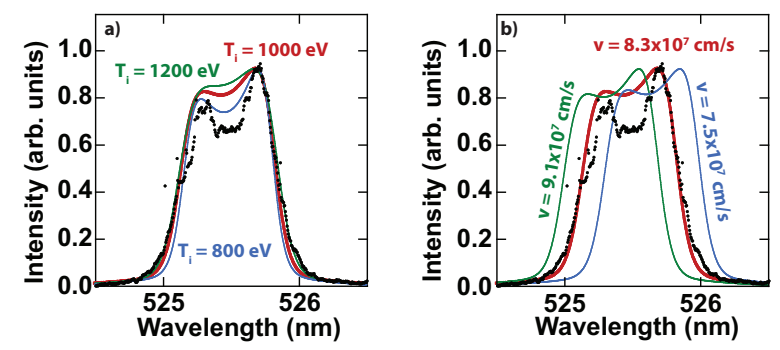

FIG. 7: (Color) The Thomson scattering cross section is fit to the measured Thomson scattering ion feature at $5.5 \mathrm{~ns}$ from a double foil target to determine the ion temperature and plasma flow velocity. The best fit to the experimental data (red line) is calculated using an electron temperature and density determined from the electron feature $(880 \mathrm{eV}$ and $1.1 \times 10^{19} \mathrm{~cm}^{-3}$ ), and ion temperature of $1000 \mathrm{eV}$ and a plasma flow velocity of $8.3 \times 10^{7} \mathrm{~cm} / \mathrm{s}$. a) The ion temperature is increased to $1200 \mathrm{eV}$ (green line) and decreased to $800 \mathrm{eV}$ (blue line) to demonstrate the sensitivity of the fit. b) The plasma flow velocity is varied from $9.1 \times 10^{7} \mathrm{~cm} / \mathrm{s}$ (green line) to $7.5 \times 10^{7} \mathrm{~cm} / \mathrm{s}$ (blue line) as well.

resulting in a factor of three increase in electron density. The electron [Fig. 9(c)] and ion [Fig. 9(d)] temperatures are also measured. The electron temperature for the double foil configuration greatly exceeds the single foil temperature for all times. The single-foil electron temperature is largest early in time and decreases for the duration of the measurements consistent with expansion cooling. The double foil electron temperature shows a distinctly different evolution, with the electron temperature increasing to over $1 \mathrm{keV}$ at $4 \mathrm{~ns}$ and then cooling. A similar trend is observed in the ion temperature measured from the double foil configuration. The ion temperature increase is slightly slower than the electron temperature increase until $3.5 \mathrm{~ns}$ at which point the ion temperature
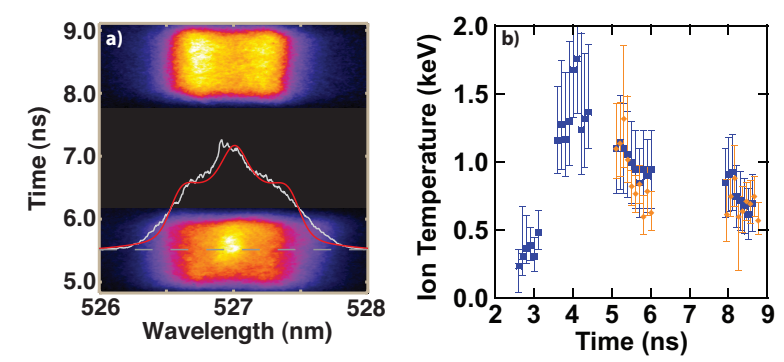

FIG. 8: (Color) a) Thomson scattering with a k-vector parallel to the target surface is shown. The experimental data at $5.5 \mathrm{~ns}$ (red line) is compared to the Thomson scattering dynamic structure factor (white line) to determine the ion temperature. b) The measured ion temperature for a k-vector normal to the target (blue squares) and parallel to the target surface (orange diamonds) is shown.
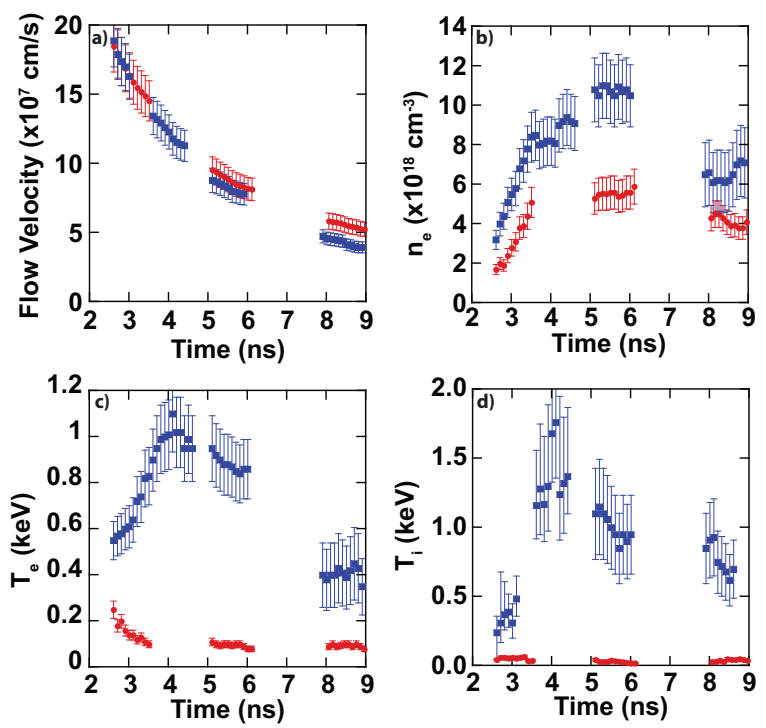

FIG. 9: (Color online) The measured flow velocity (a), electron density (b), electron temperature (c) and ion temperature (d) are shown for the double foil (blue squares) and single foil (red circles) configurations.

increases very rapidly to a temperature of $1.2 \mathrm{keV}$ exceeding the maximum measured electron temperature.

Electron-ion collisions are investigated as a possible cause of rapid electron heating in the double foil configuration. By the time significant overlap of the counterstreaming plasmas begins, flow velocities are in the range of $10^{8} \mathrm{~cm} / \mathrm{s}$. This is significantly lower than the electron thermal velocities at even a modest electron temperature of $100 \mathrm{eV}$. Therefore, we use a simple model of the electron-ion collisions where the electron scattering frequency is evaluated for the resting ions. The relative velocity of the electron gas and each of the ion streams leads to the appearance of the electron-ion drag force. The velocities of the ion streams $\mathbf{v}_{1,2}$ and the electron 


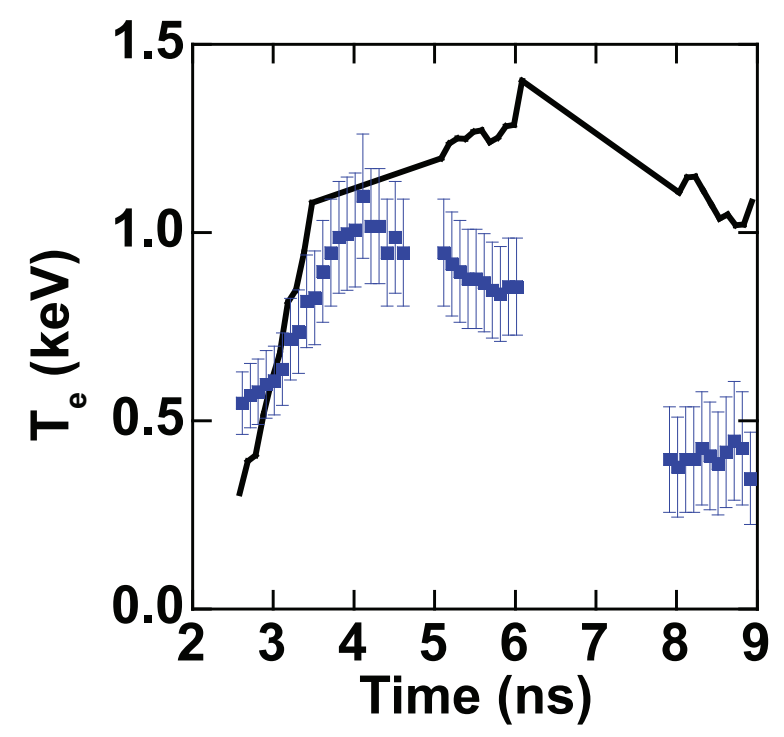

FIG. 10: (Color Online) The electron temperature from the double foil configuration (squares) is compared to Eq. 10 (black line) evaluated using the ion density inferred from the measured electron density and the measured flow velocity from the single foil configuration.

velocity $\mathbf{u}$ are related by the following equation [17],

$$
n_{e} \nu_{e i}^{(1)}\left(\mathbf{v}_{1}-\mathbf{u}\right)+n_{e} \nu_{e i}^{(2)}\left(\mathbf{v}_{2}-\mathbf{u}\right)=0
$$

where $\nu_{e i}^{(1,2)}$ are the collision frequencies between electron and ions of beams 1 and 2, which depend on the density and composition of the ion streams. We assume simple streams made of only carbon ions due to the $Z^{2}$ factors in the collision cross-sections which cause the carbon to dominant over the hydrogen. To maintain quasineutrality, $n_{e}=Z\left(n_{Z 1}+n_{Z 2}\right)$, then the force acting on the electrons from beam $1(2)$ is

$$
\mathbf{f}_{1(2)}=m_{e} n_{e} \nu_{e i}^{1(2)}\left(\mathbf{v}_{1(2)}-\mathbf{u}\right)
$$

and we assume the total force on the electrons is $\mathbf{f}_{1}+$ $\mathbf{f}_{2}=0$ due to symmetry. The presence of the friction forces leads to a slowing-down of the ions, a decrease in plasma flow velocity without a significant change in ion temperature, and heating of the electrons. The heating rate is

$$
\frac{3}{2} n_{e} \dot{T}_{e}=\mathbf{f}_{1} \cdot \mathbf{v}_{1}+\mathbf{f}_{2} \cdot \mathbf{v}_{2}=Z^{2} \eta n_{Z 1} n_{Z 2} e^{2}\left(\mathbf{v}_{2}-\mathbf{v}_{1}\right) \equiv Q
$$

using Eq. (6) and $\mathbf{f}_{1}+\mathbf{f}_{2}=0$, where $\eta=m_{e}\left(\nu_{e i}^{(1)}+\right.$ $\left.\nu_{e i}^{(2)}\right) /\left(e^{2} n_{e}\right)$. Limiting ourselves to evaluating the temperature at the mid-point of the system with symmetric plasma streams we use the following equation,

$$
\frac{\dot{T}_{e}}{T_{e}}-\frac{2}{3} \frac{\dot{n}_{e}}{n_{e}}=\frac{2}{3 n_{e} T_{e}} Q
$$

so that, according to Eq. (8),

$$
\frac{d}{d t} \frac{\left[T_{e}(t)\right]^{5 / 2}}{n_{e}^{5 / 3}}=\frac{5}{2} n_{e}^{-5 / 3}\left(\frac{2 Q T_{e}^{3 / 2}}{3 n_{e}}\right)
$$

where $Q \propto n_{e}^{2} T_{e}^{-3 / 2}$ results in the right hand side of Eq. (9) being proportional to $n_{e}^{-2 / 3}$ which can be integrated from a certain instant of time $t_{0}$ to find the temperature at any subsequent time $t$. The result reads

$$
\begin{array}{r}
{\left[T_{e}(t)\right]^{5 / 2}=\left[T_{e}\left(t_{0}\right)\right]^{5 / 2}\left(\frac{n_{Z 1}(t)}{n_{Z 2}(t)}\right)^{5 / 3}+} \\
56 \times 10^{-39} Z^{2} \Lambda_{e} n_{Z 1}^{5 / 3}(t) \int_{t_{0}}^{t}\left[n_{Z 1}\left(t^{\prime}\right)\right]^{-2 / 3} \mathbf{v}_{1}^{2}\left(t^{\prime}\right) d t^{\prime}
\end{array}
$$

where $\Lambda_{e} \approx 10$ is the Coulomb logarithm for the electronion collisions and the units are $\mathrm{cm} / \mathrm{s}$ for velocities, $\mathrm{keV}$ for temperatures, and $\mathrm{cm}^{-3}$ for densities. The measured electron temperature from the double foil is compared to Eq. 10 in Figure 10. The initial temperature and density are taken from the single foil measurements at the earliest measured time of $2.5 \mathrm{~ns}$. The ion density for a single plasma stream is assumed to be $n_{Z 1}=n_{e 1} / 6$. Equation (10) shows close agreement with the rapid increase in measured electron temperature from $2.5 \mathrm{~ns}$ until $4.0 \mathrm{~ns}$. After $4.0 \mathrm{~ns}$ the measured electron temperature is lower than the temperature predicted using Eq. (10). The most likely cause of this discrepancy is electron thermal transport which has been ignored in the development of Eq. (10). This transport would tend to reduce the electron temperature as energy is conducted away from the central region between the targets.

The rapid increase in ion temperature in the double foil configuration is an outstanding question. The electroncarbon temperature equilibration time has been calculated for the plasma conditions of interest and is found to be greater than $10 \mathrm{~ns}$ for the duration of the experiment. The directed kinetic energy of each ion stream is very large due to flow velocities greater than $10^{8} \mathrm{~cm} / \mathrm{s}$. A fraction of this directed kinetic energy is converted to electron and ion thermal energy and we are currently investigating the mechanisms involved. An assessment of ion heating by small-angle scattering, one possible ion heating mechanism, has been developed and is shown in detail in Appendix A. Equation (A14) is used with the plasma conditions from the single foil target to calculate the increase in ion temperature,

$$
T_{i}(t) \approx \frac{1.1 \times 10^{-19}}{3 L t_{0}^{2}} \int_{0}^{t_{0}} t^{3} n_{Z 1}(t) d t
$$

where $2 L$ is the distance between the targets and the hydrogen and carbon ion temperatures are assumed to be equal. The ion temperature calculated using Equation 11 is compared to the measured ion temperature in Figure 11. The measured ion temperature greatly exceeds the increase in ion temperature due to small-angle scattering 


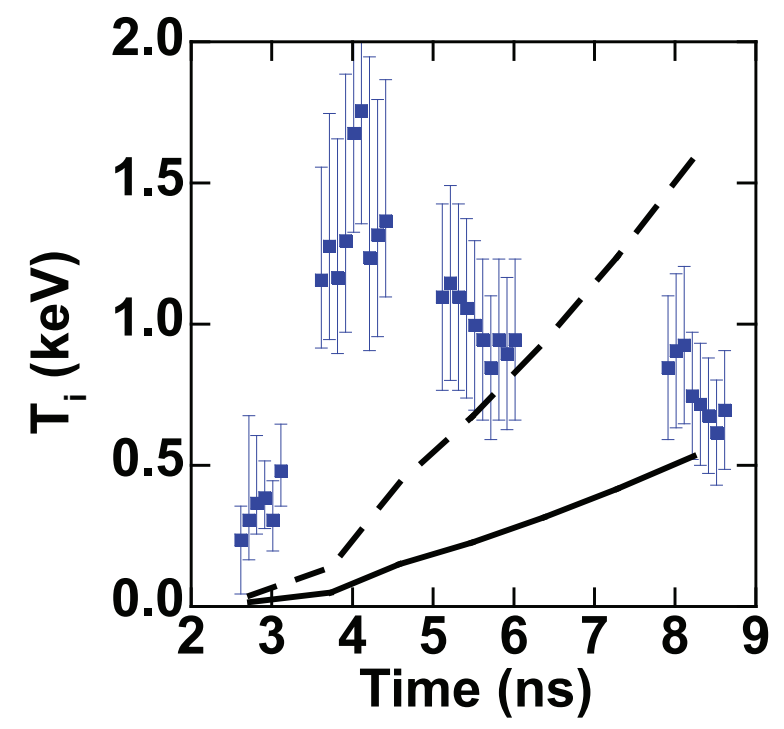

FIG. 11: (Color Online) The ion temperature from the double foil configuration (squares) is compared to Eq. 11 evaluated using the ion density inferred from the measured electron density and the measured flow velocity for the single foil configuration assuming rapid energy transfer between carbon and hydrogen ions (black line) and no energy transfer between carbon and hydrogen ions (dashed line).

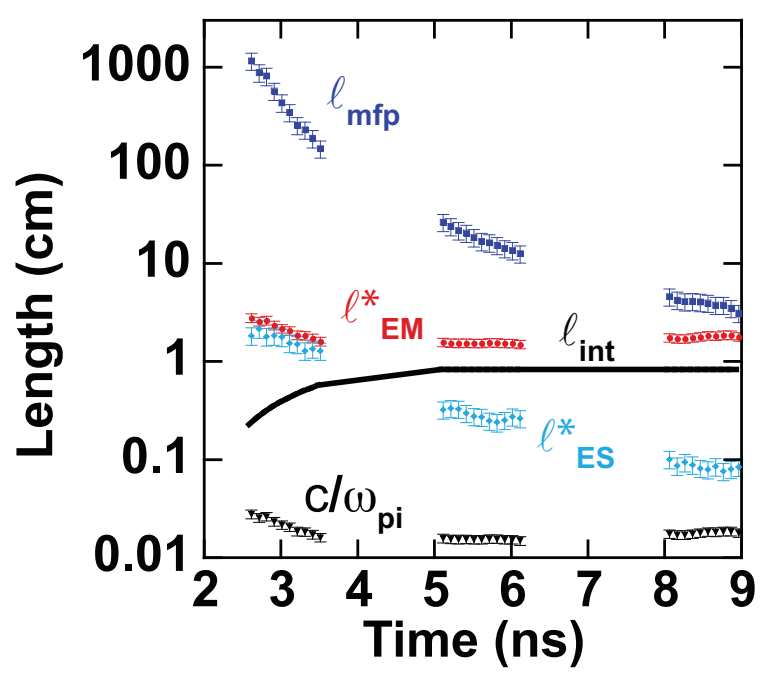

FIG. 12: (Color) The widths of the shock transition regions using Eqs. 2 (electrostatic shock, light blue points) and 3 (electromagnetic shock, red points) are compared to the measured Coulomb mean-free-path (dark blue points) and $c / \omega_{p i}$ (black points). The interaction length (black line) between the plasma flows is also shown.

for all times before 6 ns. Instabilities are being investigated as a possible source of this rapid ion heating.

In Figure 12 the measured plasma conditions from Figure 9 are used to assess the likelihood of collisionless shock formation. The Coulomb mean free path is calculated using Eq. (1) and ranges from 10 meters at 2.5 ns to $3.5 \mathrm{~cm}$ at late time. The plasma interaction length, calculated using the single foil flow velocity and limited to the distance between the foils after $5 \mathrm{~ns}$, is less than the Coulomb mean free path for all measured times. The widths of the shock transition regions are calculated using Eqs. (2) and (3) and, comparing these widths to the interaction length, it is clear that electromagnetic shocks are unlikely to develop due to a transition region that exceeds the total interaction length. An electrostatic instability, on the other hand, is possible and is one hypothesis for the rapid increase in ion temperature observed at 3.5 ns.

\section{CONCLUSIONS}

In conclusion, we have produced and characterized high velocity counter-streaming plasma flows relevant for the creation of collisionless shocks. We have measured the plasma flow velocity, electron density, and the electron and ion temperatures with high accuracy $4 \mathrm{~mm}$ from the foil surface using Thomson scattering. For the measured conditions collisionless shock formation is possible but a fully formed shock is unlikely.

Future experiments are planned for the National Ignition Facility where greatly increased laser energies will allow larger densities and flow velocities to be sustained over a longer interaction distance for longer times. By increasing the flow velocity and density significantly it will be possible to generate fully formed electromagnetic collisionless shocks.

This work was performed under the auspices of the U.S. Department of Energy by Lawrence Livermore National Laboratory under Contract DE-AC52-07NA27344 and was partially funded by the Laboratory Directed Research and Development Program under project tracking code 11-ERD-054. G. Gregori acknowledges funding from the European Research Council under the European Community's Seventh Framework Programme (FP7/2007-2013) / ERC grant agreement no. 256973.

\section{Appendix A: Ion heating by small-angle scattering}

Collisions between carbon $(\mathrm{C})$ ions are considered as a source of ion heating. The corresponding collision frequencies are much higher than those for hydrogen-carbon $(\mathrm{HC})$ and hydrogen-hydrogen $(\mathrm{HH})$ due to the $Z^{4}$ factor in the cross-section for carbon-carbon collisions. The density and velocity of the carbon ion beam propagating to the right (left) will be denoted by $n_{Z 1}\left(n_{Z 2}\right)$ and $\mathbf{v}_{Z 1}>0\left(\mathbf{v}_{Z 2}<0\right)$. Where the axis between the two beams is $x$ with $x=0$ at the midpoint between the two sources. We use here a notation $\mathrm{Z}$ (not $\mathrm{C}$ ) to generalize our discussion to ion beams other than carbon.

The ions in each stream are initially cold, but collisions between counter-propagating ion beams lead to some scattering, which gives rise to an increase in the 
ion temperature. Collisions between the ions within each stream are orders of magnitude faster as the energy of the relative ion motion for these intra-beam collisions is much smaller than the kinetic energy of each stream. Given that the ion temperatures are small, the scattering in the beam-beam collisions can be treated as though it occurred with the ions having no spread at all. Then, in the carbon-carbon collision integral describing the scattering of the beam moving in one direction on the particles of the opposite beam, the field-particle distribution can be replaced with the delta-function corresponding to the opposite beam velocity. Then, the velocity scatter for the ions moving to the right $\left(\mathbf{v}_{Z 1}>0\right)$ becomes (Eq. (6.8) in Ref. [18]):

$$
\frac{d}{d t}\left\langle\Delta \mathbf{v}^{2}\right\rangle=\frac{\beta n_{Z 2}}{2 \pi\left|\mathbf{v}_{Z 1}-\mathbf{v}_{Z 2}\right|}
$$

where

$$
\beta=\Lambda_{ \pm}\left(\frac{4 \pi Z^{2} e^{2}}{m_{Z}}\right)^{2}
$$

$\Lambda_{ \pm}$is a Coulomb logarithm for the inter-beam collisions and $m_{Z}$ is the ion mass.

As was mentioned above, the ion-ion collisions in each of the carbon beams are extremely fast, thereby maintaining a Maxwellian velocity distribution, this meaning that $\left\langle\Delta \mathbf{v}^{2}\right\rangle=3 T_{1} / m_{Z}$, where $T_{1}$ is the ion temperature of one plasma stream. In other words Eq. (A1) for the beam propagating in the positive direction can be represented as

$$
\frac{d T_{1}}{d t}=\frac{\beta n_{Z 2} m_{Z}}{6 \pi\left|\mathbf{v}_{Z 1}-\mathbf{v}_{Z 2}\right|} .
$$

For any parcel of the positive beam, Eq. (A3) allows one to find the temperature increase compared to an initial (very low) value. One would just have to substitute into Eq. (A3) the values of beam velocities and the density of the beam moving in the $-x$ direction along the path of the chosen parcel. One cannot use these equation too close to the origin of either beam, as other processes (like the electron heating by the laser pulse and a variety of associated instabilities) may occur there.

We now recall that each of the streams also contains hydrogen ions. The beam-beam collisions for them are weak, due to the smaller charge of hydrogen: if one substitutes into Eq. (A3) $m_{p}$ instead of $m_{Z}$ and replaces $Z^{4}$ by $Z^{2}$ ( $Z^{2}$ for carbon and $1^{2}$ for hydrogen) in Eq. (A2), one obtains a much weaker hydrogen heating due to hydrogen-carbon scattering. Hydrogen-hydrogen scattering is weaker still. On the other hand, the intra-beam $\mathrm{CH}$ collisions occur at a non-negligible rate and may lead to heating of the hydrogen ions (and cooling of the carbon ions). The energy transfer from carbon to hydrogen is described by equation (20.5) of Ref. [18]:

$$
\frac{d T_{H 1}}{d t}=\frac{T_{Z 1}-T_{H 1}}{\tau_{T}}
$$

$$
\tau_{T}=\frac{3 A_{Z} \sqrt{m_{p}}\left(T_{H 1}+T_{H 1} / A_{Z}\right)^{3 / 2}}{8 \sqrt{2 \pi} \Lambda Z^{2} e^{4} n_{Z 1}}
$$

The numerical expression for $\tau_{T}$ is:

$$
\tau_{T}(n s) \approx 2.1 \times 10^{20} \frac{A_{Z}}{\Lambda Z^{2}} \frac{\left(T_{H 1}+T_{H 1} / A_{Z}\right)^{3 / 2}}{n_{Z 1}}
$$

where the temperatures are measured in $\mathrm{keV}$, densities in $\mathrm{cm}^{-3}$, and velocities in $\mathrm{cm} / \mathrm{s}$.

If one wants to find the total ion thermal energy, one has from Eq. A5,

$$
\frac{d\left(T_{Z 1}+2 T_{H 1}\right)}{d t} \approx 1.8 \times 10^{-11} \frac{\Lambda n_{Z 2}}{\left|\mathbf{v}_{Z 1}-\mathbf{v}_{Z 2}\right|} .
$$

We now account for the ion cooling caused by the expansion of every parcel of the fluid in the ballistic flow, $v_{r}=r / t$. One has

$$
\nabla \cdot \mathbf{v}=\frac{1}{r^{2}} \frac{\partial r^{2} v_{r}}{\partial r}=\frac{3}{t}
$$

For an ideal monatomic gas, the cooling caused by the expansion is described by the following equation,

$$
\frac{\dot{T}}{T}=-\frac{2}{3} \nabla \cdot \mathbf{v}
$$

so that instead of Eq. (A7), we now write:

$$
\frac{d\left(T_{Z 1}+2 T_{H 1}\right)}{d t} \approx 1.8 \times 10^{-11} \frac{\Lambda n_{Z 2}}{\left|\mathbf{v}_{Z 1}-\mathbf{v}_{Z 2}\right|}-\frac{2\left(T_{Z 1}+2 T_{H 1}\right)}{t} .
$$

again with time measured in ns and temperature in $\mathrm{keV}$.

The adiabatic cooling seems to be non-negligible. To account for it in a more quantitative fashion, we would have to integrate Eq. (A10) for a parcel of fluid that arrives at the observation point at some instance $t_{0}$. The total density on axis would be $n(x, t)=n_{Z 1}(x, t)+$ $n_{Z 2}(x, t)$. Its positions prior to that having been $x=$ $L\left(t / t_{0}-1\right)$, and one can show that the density seen by the chosen parcel is,

$$
n_{Z 1}(x, t)=\frac{1}{\left(2-t / t_{0}\right)^{2} n_{1}\left(\frac{t}{2-t / t_{0}}\right)} .
$$

where $n_{1}$ is the density of one plasma stream measured at the mid point. The velocity difference that also enters is

$$
\mathbf{v}_{Z 1}-\mathbf{v}_{Z 2}=\frac{2 L}{t}
$$

which yields the following differential equation for $\mathrm{T}$ :

$$
\frac{d\left(T_{Z 1}+2 T_{H 1}\right)}{d t}+\frac{2\left(T_{Z 1}+2 T_{H 1}\right)}{t} \approx 1.1 \times 10^{-19} \frac{t n_{Z 2}}{L} .
$$

where $L$ is measured in $\mathrm{cm}, t$ in ns, $n$ in $\mathrm{cm}^{-3}$ and $T$ in $\mathrm{keV}$. This equation can be integrated:

$$
T_{Z 1}(t)+2 T_{H 1}(t) \approx \frac{1.1 \times 10^{-19}}{L t_{0}^{2}} \int_{0}^{t_{0}} t^{3} n_{Z 2}(t) d t .
$$


Now, plugging in the experimental dependence of the plasma stream density vs. time, one can find the col- lisional prediction of the ion temperature.

L. Gargate, et al., High Energy Density Physics pp. (2011).

[11] R. P. Drake, D. Phillion, K. Estabrook, R. Turner, R. Kauffman, and E. Campbell, Phys Fluids B-Plasma 1, 1089 (1989).

[12] J. A. Fejer, Canadian Journal of Physics 38, 1114 (1960).

[13] J. Sheffield, Plasma Scattering of Electromagnetic Radiation (Academic, New York, 1975).

[14] I. H. Hutchinson, Principles of Plasma Diagnostics (Cambridge, 1987).

[15] S. H. Glenzer, C. A. Back, L. J. Suter, M. A. Blain, O. L. Landen, J. D. Lindle, B. J. MacGowan, G. F. Stone, R. E. Turner, and B. H. Wilde, Physical Review Letters 79, 1277 (1997).

[16] D. H. Froula, L. Divol, and S. H. Glenzer, Physical Review Letters 88, 105003 (2002).

[17] D. Ryutov, N. Kugland, H. Park, S. Pollaine, B. Remington, and J. Ross, Physics Of Plasmas 18 (2011).

[18] B. A. Trubnikov, Reviews of Plasma Physics 1, 105 (1965).

[10] H.-S. Park, D. Ryutov, J. Ross, N. Kugland, S. Glenzer, C. Plechaty, S. Pollaine, B. Remington, A. Spitkovsky, 